\title{
COMPACT PERTURBATIONS OF CERTAIN VON NEUMANN ALGEBRAS
}

BY

JOAN K. PLASTIRAS

\begin{abstract}
Let $\delta$ be a sequence of mutually orthogonal, finite dimensional projections whose sum is the identity on a Hilbert space $\mathcal{X}$. If we denote the commutant of $\mathcal{E}$ by $\mathscr{D}(\mathcal{E})$ and the ideal of compact operators on $\mathcal{X}$ by $\mathcal{C}(\mathcal{F})$, then it is easily verified that $\mathscr{D}(\mathcal{E})+\mathcal{C}(\mathcal{H})=\{T+K: T \in \mathscr{D}(\mathcal{E})$, $K \in \mathcal{C}(\mathcal{H})$ \} is a $C^{*}$-algebra. In this paper we classify all such algebras up to *-isomorphism and characterize them by examining their relationship to certain quasidiagonal and quasitriangular algebras.
\end{abstract}

Throughout this paper, $\mathcal{E}$ will denote a sequence $\left\{E_{i}\right\}_{i=1}^{\infty}$ of mutually orthogonal, finite dimensional projections on a Hilbert space whose sum is the identity. The set consisting of every bounded operator whose matrix representation is diagonal with respect to $\mathscr{E}$ will be referred to as the block diagonal algebra associated with $\mathcal{E}$ and denoted as $\mathscr{D}(\mathcal{E})$; we remark that $\mathscr{D}(\mathcal{E})$ can be viewed as the commutant of $\mathcal{E}$, or as the direct product of the von Neumann algebras $\mathcal{L}\left(E_{i} \mathcal{H}\right)$, where $\mathcal{L}(\mathcal{H})$ (respectively $C(\mathcal{H})$ ) will denote the algebra of bounded (respectively compact) operators on a separable Hilbert space.

Definition. $\mathscr{D}(\mathcal{E})+\mathcal{C}(\mathcal{H})=\{T+K: T \in \mathscr{D}(\mathcal{E}), K \in \mathcal{C}(\mathcal{H})\}$ will be referred to as the perturbed block diagonal algebra for $\mathcal{E}$.

By $\left[2\right.$, Chapter 1], $\mathscr{D}(\mathscr{E})+\mathcal{C}(\mathcal{F})$ is a $C^{*}$-algebra. It is weak operator dense in $\mathcal{L}(\mathcal{H})$ because $\mathcal{C}(\mathcal{H})$ is and is not norm separable because $\mathscr{D}(\mathcal{E})$ is not. It is not hard to realize that the structure of such an algebra depends only on the dimensions of the projections in $\mathcal{E}$, not on how we label them, so we will occasionally suppress $\mathcal{E}$ and wirte $\mathscr{D}\left\{\left(\alpha_{i}\right)_{i=1}^{\infty}\right\}+\mathcal{C}(\mathcal{H C})$ instead, where $\alpha_{i}$ is the dimension of $E_{i}$ for each $i$.

One might guess that two perturbed block diagonal algebras are isomorphic if their associated sequences agree after a finite number of terms. Indeed, $\mathscr{D}\{(2,1,1,1, \ldots)\}+.\mathcal{C}(\mathcal{H C})$ is isomorphic to $\mathscr{D}\{(1,1,1,1, \ldots)\}+\mathcal{C}(\mathcal{H C})$. But

Received by the editors February 18, 1976 and, in revised form, June 10, 1976.

AMS (MOS) subject classifications (1970). Primary 47A55, 47B05, 47C10; Secondary 46L05, 47A65.

Key words and phrases. Perturbed block diagonal algebras, $C^{*}$-algebra isomorphism, compact perturbations, quasidiagonal operators, Fredholm index, Hilbert space.

- American Mathematical Society 1977 
what is perhaps surprising is that $\mathscr{D}\{(1,2,2,2, \ldots)\}+C(\mathscr{H})$ is not isomorphic to $\mathscr{D}\{(2,2,2,2, \ldots)\}+\mathcal{C}(\mathcal{H})$.

THEOREM 1. Let $\mathbb{Q}=\mathscr{D}(\mathscr{E})+\mathcal{C}(\mathcal{H})$ and $\mathscr{B}=\mathscr{D}(\mathscr{F})+\mathcal{C}(\mathcal{H})$. $\mathbb{Q}$ is isomorphic to $B$ if and only if there exist finite subsets of the positive integers, $N_{0}$ and $N_{1}$, and a bijection $\tau: \mathbf{N}-N_{0} \rightarrow \mathbf{N}-N_{1}$ such that

$\operatorname{dim}\left(\sum_{i \in N_{0}} E_{i}\right)=\operatorname{dim}\left(\sum_{j \in N_{1}} F_{j}\right)$ and $\operatorname{dim} E_{i}=\operatorname{dim} F_{\tau(i)} \quad$ for all $i \in \mathbf{N}-N_{0}$.

We include a brief discussion of the important ideas in the proof of Theorem 1. That the condition is sufficient to insure isomorphism follows from the construction of a unitary operator $U$ such that

$$
U\left(\sum_{i \in N_{0}} E_{i}\right) U^{*}=\sum_{i \in N_{1}} F_{i} \text { and } U E_{i} U^{*}=F_{\tau(i)} \text { for all } i \text { in } \mathbf{N}-N_{0},
$$

and the realization that $U$ implements an isomorphism from $\mathcal{Q}$ to $\mathscr{B}$. That the condition is necessary to insure isomorphism is less obvious; the main technical effort is contained in the following lemma:

Lemma 2. Any selfadjoint unitary operator $U$ which implements an automorphism of a perturbed block diagonal algebra $\mathscr{D}(\mathcal{E})+\mathcal{C}(\mathcal{H})$ is a compact perturbation of a selfadjoint unitary operator $V$ which implements an automorphism of $\mathscr{D}(\mathcal{E})$.

Assuming the validity of Lemma 2, we sketch the remainder of the proof as follows:

Proof of Theorem 1. If $\mathbb{Q}$ and $\mathscr{B}$ are the isomorphic algebras of Theorem 1 , we let

$$
C=\left\{\left(\begin{array}{ll}
T_{11} & T_{12} \\
T_{21} & T_{22}
\end{array}\right): T_{11} \in \mathcal{Q}, T_{22} \in \mathscr{B} ; T_{21}, T_{12} \in \mathcal{C}(\mathcal{H C})\right\} .
$$

It is easily verified that $C$ is a perturbed block diagonal algebra on $\mathscr{K}=\mathscr{K}$ $\oplus \mathcal{K}$; i.e., $C=\mathscr{D}(\mathscr{E}) \oplus \mathscr{D}(\mathscr{F})+\mathcal{C}(\mathcal{K})$. It follows from [8, Lemma 4.5] that any isomorphism $\alpha$ between $\mathcal{Q}$ and $\mathscr{B}$ is implemented by a unitary operator $W$. We then remark that the unitary operator $U$, whose representation as a

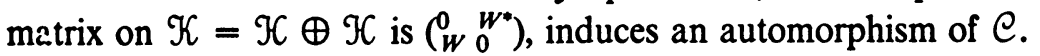

After noting that $U$ is selfadjoint, we apply Lemma 2 to produce a selfadjoint operator $V$ which implements an automorphism of $\mathscr{D}(\mathscr{E}) \oplus \mathscr{D}(\mathscr{F})$ and such that $V=U+Q$, where $Q$ is a compact operator on $\mathcal{K}$ and is selfadjoint because $V$ and $U$ are. Hence,

$$
V=U+\left(\begin{array}{ll}
Q_{11} & Q_{12} \\
Q_{21} & Q_{22}
\end{array}\right)=\left(\begin{array}{cc}
Q_{11} & Q_{12}+W^{*} \\
Q_{21}+W & Q_{22}
\end{array}\right)
$$


Since $V$ implements an automorphism of $\mathscr{D}(\mathscr{E}) \oplus \mathscr{D}(\mathscr{F})$ it shuffles the projections in $\mathcal{E} \cup \mathcal{F}$ and since $V$ is selfadjoint, we conclude that there exist subsets $N_{0}$ and $N_{1}$ of $\mathbf{N}$ and bijections:

$$
\begin{gathered}
\tau_{0}: N_{0} \rightarrow N_{0} \text { such that } V E_{i} V^{*}=E_{\tau_{0}(i)} \text { for all } i \text { in } N_{0}, \\
\tau_{1}: N_{1} \rightarrow N_{1} \text { such that } V F_{j} V^{*}=F_{\tau_{1}(j)} \text { for all } j \text { in } N_{1} \text {, and } \\
\tau: \mathbf{N}-N_{0} \rightarrow \mathrm{N}-N_{1} \text { such that } V E_{i} V^{*}=F_{\tau(i)} \text { for all } i \text { in } \mathbf{N}-N_{0} .
\end{gathered}
$$

Since $V$ is unitary, $\operatorname{dim}\left(E_{i}\right)=\operatorname{dim}\left(V E_{i} V^{*}\right)=\operatorname{dim}\left(F_{\tau(i)}\right)$ for all $i \in \mathbf{N}-N_{0}$, so it remains only to show that $N_{0}$ is a finite subset of $\mathbf{N}$ and that $\operatorname{dim}\left(\Sigma_{i \in N_{0}} E_{i}\right)=\operatorname{dim}\left(\sum_{j \in N_{1}} F_{j}\right)$. One can conclude from the matrix representation of $V$ that $Q_{11}$ is a selfadjoint partial isometry and that $\Sigma_{i \in N_{0}} E_{i}$ is the projection onto the initial space of $Q_{11}$ while $\Sigma_{j \in N_{1}} F_{j}$ is the projection onto the initial space of $Q_{22}(*)$.

Since $Q_{11}$ is compact, we deduce immediately that $N_{0}$ is finite. Note, also, that the initial space of $Q_{11}$ is the kernel of $Q_{21}+W$. Since $Q_{21}+W$ is a compact perturbation of a unitary operator, the index of $Q_{21}+W$ is zero, implying that

$$
\operatorname{dim} \operatorname{ker}\left(Q_{21}+W\right)=\operatorname{dim} \operatorname{ker}\left(Q_{21}+W\right)^{*}=\operatorname{dim} \operatorname{ker}\left(Q_{12}+W^{*}\right),
$$

with the last equality following from the fact that $Q$ is selfadjoint. Since the kernel of $Q_{12}+W^{*}$ is the initial space of $Q_{22}$, we conclude that the dimension of the initial space of $Q_{11}$ equals the dimension of the initial space of $Q_{22}$. It follows from (*) that $\operatorname{dim}\left(\sum_{i \in N_{0}} E_{i}\right)=\operatorname{dim}\left(\sum_{j \in N_{1}} F_{j}\right)$, thus concluding the proof of Theorem 1 .

We now proceed (from Proposition 3 through Lemma 9) to gather the facts necessary to verify Lemma 2.

PROPOSITION 3. Let $\mathcal{F}=\left\{F_{n}\right\}_{n=1}^{\infty}$ be any sequence of finite dimensional, mutually orthogonal projections. Then $\left\|K F_{n}\right\| \rightarrow 0$ for every compact operator $K$.

Proof. Arguing as in [3, Lemma 1, p. 292] this is easily verified for the finite rank operators and because these are norm-dense in $\mathcal{C}(\mathcal{H})$, the assertion follows by an application of the triangle inequality.

Definition. Let $\delta_{\mathscr{E}}$ denote the map which takes an operator $T$ to the formal sum $\delta_{\mathcal{E}}(T)=\sum_{k=1}^{\infty} E_{k} T E_{k}$, by which we mean the strong limit of $\sum_{k=1}^{n} E_{k} T E_{k}$. Since

$$
\left\|\delta_{\mathscr{E}}(T)\right\|=\left\|\sum_{k=1}^{\infty} E_{k} T E_{k}\right\|=\sup _{k}\left\|E_{k} T E_{k}\right\| \leqslant\|T\| \text { and }\left\|\delta_{\mathcal{E}}(1)\right\|=\|1\|,
$$

$\delta_{\mathcal{E}}$ is a linear operator on $\mathcal{L}(\mathcal{H})$ of norm one.

Proposition 4. Suppose $T \in \mathscr{D}(\mathcal{E})+\mathcal{C}(\mathcal{H})$. Then $T-\delta_{\mathscr{E}}(T)$ is compact.

Proof. From the definition of $\mathscr{D}(\mathcal{E})+\mathcal{C}(\mathcal{H}), T=S+K$, where $S \in$ 
$\mathscr{D}(\mathscr{E})$ and $K \in \mathcal{C}(\mathcal{H C})$. So, $\delta_{\mathscr{\delta}}(T)=\delta_{\mathscr{E}}(S+K)=S+\delta_{\mathscr{\delta}}(K)=T-K+$ $\delta_{\mathscr{E}}(K)$ and $T-\delta_{\mathscr{E}}(T)=K-\delta_{\mathscr{E}}(K)$. It follows that $T-\delta_{\mathscr{E}}(T)$ is compact because $K$ and $\delta_{\delta}(K)$ are.

LEMMA 5. Assume that $U$ is a unitary operator which implements an automorphism of $\mathscr{D}(\mathcal{E})+\mathcal{C}(\mathcal{H C})$. Then $\lim _{i} \inf _{j}\left\|E_{j}^{\perp} U E_{i}\right\|=0$.

Proof. In this contrapositive argument we assume that $\inf _{j}\left\|E_{j}^{\perp} U E_{i}\right\| \nrightarrow 0$ and then show that $U$ does not implement an automorphism of $D(\mathcal{E})+$ $\mathcal{C}(\mathcal{F})$. So there exist $\varepsilon>0$ and an infinite subset $M$ of $\mathbf{N}$ such that $\inf _{j \in \mathrm{N}}\left\|E_{j}{ }^{\perp} U E_{i}\right\|>\varepsilon$ for all $i \in M(*)$. We will inductively obtain a set of finite dimensional, mutually orthogonal projections $\left\{C_{i_{k}}, D_{i_{k}}\right\}_{k=1}^{\infty}$ for which $\left\|C_{i_{k}} U E_{i_{k}}\right\|$ and $\left\|D_{i_{k}} U E_{i_{k}}\right\|$ are both greater than $\varepsilon / 4$. Let $i_{1}$ be the first integer in $M$. There are two possibilities:

(1) If $\max _{j}\left\|E_{j} U E_{i_{1}}\right\|>\varepsilon / 4$ then let $j_{1}$ be any integer such that $\left\|E_{j_{1}} U E_{i_{1}}\right\|>$ $\varepsilon / 4$. By (*) it follows that $\left\|\Sigma_{j \neq j_{1}} E_{j} U E_{i_{1}}\right\| \geqslant \varepsilon$ and since $\sum_{j=1, j \neq j_{1}}^{n} E_{j} U E_{i_{1}}$ tends strongly to $\Sigma_{j \neq j_{1}} E_{j} U E_{i_{1}}$, it follows that for some $n_{1} \in N,\left\|\sum_{j \neq j_{1}}^{n_{1}} E_{j} U E_{i_{i}}\right\|>$ $\varepsilon / 4$. Let $C_{i_{1}}=E_{j_{1}}$ and $D_{i_{1}}=\sum_{j=1, j \neq j_{1}}^{n_{1}} E_{j}$.

(2) If $\max _{j}\left\|E_{j} U E_{i_{1}}\right\|<\varepsilon / 4$, let $n_{1}$ be the first integer for which $\left\|\sum_{j=1}^{n_{1}} E_{j} U E_{i_{1}}\right\|>\varepsilon / 4$. Then

$$
\left\|\sum_{j=1}^{n_{1}} E_{j} U E_{i_{1}}\right\|<\left\|\sum_{j=1}^{n_{1}-1} E_{j} U E_{i_{1}}\right\|+\left\|E_{j_{1}} U E_{i_{1}}\right\|<\varepsilon / 2 .
$$

Hence $\left\|\sum_{j=n_{1}+1}^{\infty} E_{j} U E_{i_{1}}\right\|>\varepsilon / 2$ because $\left\|U E_{i_{1}}\right\|=1>\varepsilon$ and so there exists $n_{2}$ such that $\left\|\sum_{j=n_{1}+1}^{n_{2}} E_{j} U E_{i_{1}}\right\|>\varepsilon / 4$. Let $C_{i_{1}}=\sum_{j-1}^{n_{1}} E_{j}$ and $D_{i_{1}}=\sum_{j=n_{1}+1}^{n_{2}} E_{j}$.

For the $k$ th inductive step, let $i_{k}$ be the first integer in $M$ such that $\left\|\left(\sum_{j=1}^{k-1} C_{i j}+D_{i j}\right) U E_{i_{k}}\right\|<\varepsilon / 8$. That such an integer exists follows from Proposition 3 and the fact that $\left(\sum_{j=1}^{k-1} C_{i j}+D_{i j}\right) U$ is compact. Since $\|\left(\sum_{j=1}^{k-1} C_{i_{j}}+\right.$ $\left.D_{i}\right)^{\perp} U E_{i_{k}} \|>7 \varepsilon / 8$, the reader can verify that we are able to repeat the process to obtain mutually orthogonal, finite dimensional subprojections of $\left(\sum_{j=1}^{k-1} C_{i_{j}}+D_{i_{j}}\right)^{\perp}, C_{i_{k}}$ and $D_{i_{k}}$, such that $\left\|C_{i_{k}} U E_{i_{k}}\right\|,\left\|D_{i_{k}} U E_{i_{k}}\right\|>\varepsilon / 4$.

We now pass to a subsequence $\left\{h_{k}\right\}$ of $\left\{i_{k}\right\}$ such that for every $k \in \mathbf{N}$,

$$
\sum_{l \neq k}\left\|C_{h_{l}} U E_{h_{k}}\right\| \cdot\left\|D_{h_{l}} U E_{h_{k}}\right\|<\varepsilon^{2} / 32
$$

To do so, let $\left\{\alpha_{i, j}\right\}_{i j \in N}$ be a set of positive real numbers for which $\Sigma_{i, j \in N} \alpha_{i j}^{2}<$ $\varepsilon^{2} / 32$. Let $h_{1}=i_{1}$. Assuming that we have obtained $\left\{h_{k}\right\}_{k=1}^{n}$, let $h_{n+1}$ be the next integer in $\left\{i_{k}\right\}$ such that for every $l<n+1,\left\|C_{h_{n+1}} U E_{h_{1}}\right\|$ and $\left\|D_{h_{n+1}} U E_{h_{l}}\right\|$ are each less than $\alpha_{n+1, l}$, whereas $\left\|E_{h_{l}} U E_{h_{n+1}}\right\|$ and $\left\|D_{h_{1}} U E_{h_{n+1}}\right\|$ are each less than $\alpha_{l, n+1}$; this is possible by Proposition 3 applied to $U E_{h}$, $C_{h_{l}} U$, and $D_{h_{l}} U$ for those $l \Varangle n+1$. Continue inductively.

We will construct an operator $T \in \mathscr{D}(\mathcal{E})$ such that $U T U^{*} \notin \mathscr{D}(\mathcal{E})+$ 
$\mathcal{C}(\mathcal{H})$, thus concluding the argument. Since $\left\|C_{h_{k}} U E_{h_{k}}\right\|$ and $\left\|E_{h_{k}} U^{*} D_{h_{k}}\right\|$ are both greater than or equal to $\varepsilon / 4$, there is a rank one partial isometry $T_{h_{k}} \in \mathcal{L}\left(E_{h_{k}} \mathcal{H}\right)$ for which $\left\|C_{h_{k}} U E_{h_{k}} T_{h_{k}} E_{h_{k}} U^{*} D_{h_{k}}\right\| \geqslant \varepsilon^{2} / 16$ and we let $T=$ $\Sigma_{k \in N} T_{h_{k}}$. For arbitrary $l \in \mathbf{N}$,

$$
C_{h_{l}} U T U^{*} D_{h_{l}}=C_{h_{l}} U T_{h_{l}} U^{*} D_{h_{l}}+\sum_{k \neq 1} C_{h_{l}} U T_{h_{k}} U^{*} D_{h_{l}}
$$

Hence,

$$
\begin{aligned}
\left\|C_{h_{l}} U T U^{*} D_{h_{l}}\right\| & \geqslant\left\|C_{h_{l}} U T_{h_{l}} U^{*} D_{h_{l}}\right\|-\left\|\sum_{k \neq 1} C_{h_{l}} U T_{h_{k}} U^{*} D_{h_{l}}\right\| \\
& \geqslant\left\|C_{h_{l}} U T_{h_{l}} U^{*} D_{h_{l}}\right\|-\sum_{k \neq l}\left\|C_{h_{l}} U E_{h_{k}}\right\|\left\|D_{h_{l}} U E_{h_{k}}\right\| \\
& \geqslant \frac{\varepsilon^{2}}{16}-\frac{\varepsilon^{2}}{32}=\frac{\varepsilon^{2}}{32} .
\end{aligned}
$$

So, $\lim _{l}\left\|C_{h_{l}}\left(U T U^{*}-\delta_{\mathscr{E}}\left(U T U^{*}\right)\right) D_{h_{l}}\right\|=\lim _{l}\left\|C_{h_{l}} U T U^{*} D_{h_{l}}\right\|>\varepsilon^{2} / 32$ implies that $\overline{U T} U^{*}-\delta_{\mathscr{E}}\left(U T U^{*}\right)$ is not compact by Proposition 3 and therefore that $U T U^{*}$ does not belong to $\mathscr{D}(\mathcal{E})+\mathcal{C}(\mathcal{H})$ by Proposition 4 . This concludes the argument.

Lemma 6. Assume that $P$ and $Q$ are projections. Then

$$
\|P-Q\|=\max \left\{\left\|P^{\perp} Q\right\|,\left\|P Q^{\perp}\right\|\right\} .
$$

Proof. Since $P-Q=P Q^{\perp}-P^{\perp} Q$, it follows that $\|P-Q\|=\| P Q^{\perp}$ - $P^{\perp} Q \|$. Because $P Q^{\perp}$ and $P^{\perp} Q$ have orthogonal initial and final spaces,

$$
\left\|P Q^{\perp}-P^{\perp} Q\right\|=\max \left\{\left\|P Q^{\perp}\right\|,\left\|P^{\perp} Q\right\|\right\} .
$$

LEMMA 7. If $U$ is a selfadjoint unitary operator which implements an automorphism of $\mathscr{D}(\mathcal{E})+\mathcal{C}(\mathcal{H C})$ then there exists a bijection $\sigma$ of $\mathbf{N}$ such that $\| U E_{l} U^{*}$ $-E_{\sigma(i)} \| \rightarrow 0$.

Proof. From Lemma 5 there exists a subset $M_{0}$ of $\mathbf{N}$ such that $\mathbf{N}-M_{0}$ is

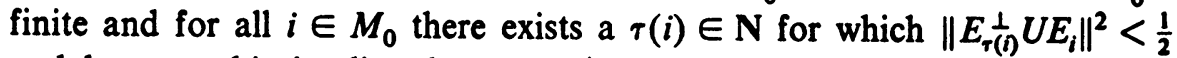
and because this implies that $\left.E_{\tau(i)} U\right|_{E_{1} x}$ has no null space it follows that $\operatorname{dim} E_{\tau(i)}>\operatorname{dim} E_{i}$; that $\operatorname{dim} E_{\tau(i)}<\operatorname{dim} E_{i}$ for all but finitely many $i \in M_{0}$ follows from an argument similar to that in Lemma 5 which we include for completeness:

Contrapositively, we assume that $\operatorname{dim} E_{\tau(i)}>\operatorname{dim} E_{i}$ for all $i$ in an infinite subset $S$ of $M_{0}$. Let $i_{1}$ be the first integer in $S$. Since $U$ is unitary there is an integer $n_{1}$ such that

$$
\left\|\left(E_{\tau\left(i_{1}\right)} \ominus \operatorname{ran} U E_{i_{1}}\right) U\left(\sum_{l=1, l \neq i_{1}}^{n_{1}} E_{l}\right)\right\|>\frac{1}{4}
$$


whereas, since $i_{1} \in M_{0},\left\|E_{\tau\left(i_{1}\right)} U E_{i_{1}}\right\|>\frac{1}{4}$. Because $U=U^{*}$, $\left\|\left(\sum_{l=1 ; l \neq i_{1}}^{n_{1}} E_{l}\right) U E_{\tau\left(i_{1}\right)}\right\|,\left\|E_{i_{1}} U E_{\tau\left(i_{1}\right)}\right\| \geqslant \frac{1}{4}$. Assuming that you have selected $\left\{i_{l}\right\}_{l=1}^{k-1}$, let $i_{k}$ be the next integer in $S$ such that $i_{k}>n_{k-1}$ and $\left\|E_{\tau\left(i_{k}\right)} U\left(\sum_{l=1}^{n_{k}-1} E_{l}+\sum_{l=1}^{k-1} E_{i}\right)\right\|<\frac{1}{4}$. There exists an integer $n_{k}$ such that

$$
\left\|\left(E_{\tau\left(i_{k}\right)} \ominus \operatorname{ran} U E_{i_{k}}\right) U\left(\sum_{l=n_{k-1}+1, l \neq i_{k}}^{n_{k}} E_{l}\right)\right\| \geqslant \frac{1}{4} .
$$

Continue inductively to obtain an infinite set of finite dimensional, mutually orthogonal projections $\left\{C_{i_{k}}, D_{i_{k}}\right\}$ where $C_{i_{k}}=\sum_{l=n_{k-1}+1}^{n_{k}} E_{l}$ and $D_{i_{k}}=E_{i_{k}}$ so that $\left\|C_{i_{k}} U E_{\tau\left(i_{k}\right)}\right\|,\left\|D_{i_{k}} U E_{\tau\left(i_{k}\right)}\right\| \geqslant \frac{1}{4}$ and apply the argument of Lemma 5 to arrive at a contradiction-thereby forcing $\operatorname{dim} E_{\tau(i)}=\operatorname{dim} E_{i}$ for all but finitely many $i \in M_{0}$. If we let $M=\left\{i \in M_{0}: \operatorname{dim} E_{i}=\operatorname{dim} E_{\tau(i)}\right\}$ then we conclude that $\mathbf{N}-M$ is finite.

We make the following simple observation: if $E$ and $F$ are projections and $U$ is unitary, then for $x \in E \mathcal{T C}$ with $\|x\|=1,\|F U E x\|^{2}+\left\|F^{\perp} U E x\right\|^{2}=1$ so that

$$
1-\inf _{\substack{x \in E \mathcal{C} \\\|x\|=1}}\|F U E x\|^{2}=\sup _{\substack{x \in E \mathcal{X} \\\|x\|=1}}\left\|F^{\perp} U E x\right\|^{2}=\left\|F^{\perp} U E\right\|^{2} \quad(*) .
$$

Next we observe that since $\left.E_{\tau(i)} U\right|_{E_{i} \mathcal{X}} \in \mathcal{L}\left(E_{i} \mathcal{T} \mathcal{C}, E_{\tau(i)} \mathcal{S C}\right)$ is invertible, $\left.E_{\tau(i)} U\right|_{E_{i} \mathcal{X}}=V P$ where $P \in \mathcal{L}\left(E_{i} \mathcal{T C}\right)$ is positive and $V \in \mathcal{L}\left(E_{i} \mathcal{H}, E_{\tau(i)} \mathcal{H C}\right)$ is unitary by the polar decomposition; since $U=U^{*},\left.E_{i} U\right|_{E_{i}(9)}=P V^{*}$. We use this fact to show that $\left\|E_{\tau(i)} U E_{i}^{\perp}\right\|=\left\|E_{\tau(i)}^{\perp} U E_{i}\right\|$ for all $i \in M$ :

$$
\begin{aligned}
& \left\|E_{\tau(i)} U E_{i}^{\perp}\right\|^{2}=\left\|E_{i}{ }^{\perp} U E_{\tau(i)}\right\|^{2}=\sup _{\substack{\left.x \in E_{\tau^{(i)}}\right) \mathcal{c} \\
\|x\|=1}}\left\|E_{i}{ }^{\perp} U E_{\tau(i)} x\right\|^{2} \quad \text { because } U=U^{*} \\
& =1-\inf _{\left.x \in E_{\tau(i)}\right)}\left\|E_{i} U E_{\tau(i)} x\right\|^{2} \quad \text { by }(*) \\
& \|x\|=1 \\
& =1-\inf _{\substack{x \in E_{z}(i) x \\
\|x\|=1}}\left\|P V^{*} x\right\|^{2}=1-\inf _{\substack{y \in E_{i}, x \\
\|y\|=1}}\|P y\|^{2} \quad \text { because } V^{*} \text { is unitary } \\
& =1-\inf _{\substack{y \in E_{i} \Im \mathcal{C} \\
\|y\|=1}}\|V P y\|^{2}=1-\inf _{\substack{y \in E_{i} \mathcal{N} \\
\|y\|=1}}\left\|E_{\tau(i)} U E_{i} y\right\|^{2}=\left\|E_{\tau(i)}^{1} U E_{i}\right\|^{2} \quad \text { by (*). }
\end{aligned}
$$

From the construction of $M$ and Lemma 6 we conclude that

$$
\left\|U E_{i} U^{*}-E_{\tau(i)}\right\|=\max \left\{\left\|E_{\tau(i)}^{\perp} U E_{i}\right\|,\left\|E_{\tau(i)} U E_{i}{ }^{\perp}\right\|\right\} \rightarrow 0 .
$$

We let $\sigma(i)=\tau(i)$ for all $i \in M$ and let $\sigma(i)=i$ for all $i \notin M$ so that to obtain the result it remains only to show that $\tau$ is a bijection of $M$. But, for each $i \in M$, 


$$
\begin{aligned}
\frac{1}{2}>\left\|U E_{i} U-E_{\tau(i)}\right\| & =\left\|U^{2} E_{i} U^{2}-U E_{\tau(i)} U\right\|=\left\|E_{i}-U E_{\tau(i)} U\right\| \\
& =\max \left\{\left\|E_{i}{ }^{\perp} U E_{\tau(i)}\right\|,\left\|E_{i} U E_{\tau(i)}^{\perp}\right\|\right\}
\end{aligned}
$$

so that $\operatorname{dim} E_{i}=\operatorname{dim} U E_{\tau(i)} U=\operatorname{dim} E_{\tau(i)}$ and $\left\|E_{i}^{\perp} U E_{\tau(i)}\right\|<\frac{1}{2}$. We remark that $\tau^{2}(i)=i$ for all $i$ in $M$ because the value of $\tau$ is uniquely determined since $\|U\|=1$ and $\inf _{x \in E_{i} Y Y_{i}\|x\|=1}\left\|E_{\tau(i)} U E_{i} x\right\|^{2}>\frac{1}{2}$ by (*). Hence, $\tau$ is a one-to-one map of $M$ onto $M$, thus concluding our argument.

Construction 8. Suppose $U$ is a selfadjoint unitary operator which implements an automorphism of $\mathscr{D}(\mathscr{E})+C(\mathcal{F})$. From $U$ we will construct a selfadjoint unitary $V$ which implements an automorphism of $\mathscr{D}(\mathcal{E})$ such that $\left\|U E_{j} U^{*}-V E_{j} V^{*}\right\| \rightarrow 0$.

Proof. Let $M$ be the set and $\sigma$ the bijection of $\mathbf{N}$ introduced in Lemma 7. To define $V \sim\left(V_{i j}\right)$, let $V_{i j} \equiv 0$ whenever $i \neq \sigma(j)$ and let $V_{\sigma()_{j} j}=1$ whenever $j \notin M$. For $j \in M$ recall that $\operatorname{dim} E_{j}=\operatorname{dim} E_{o(j)}$ so that $\left.E_{o(j)} U\right|_{E, X}$ can be identified as the matrix of an operator from one $n$-dimensional Hilbert space to another with polar decomposition $W_{\sigma(), j} P_{\sigma(), j}$; since $\left.E_{\sigma(j)} U\right|_{E, j C}$ is invertible for $j \in M, W_{o(j) j}$ is unitary. We proceed, inductively, as follows: Suppose $j_{1}$ is the first integer in $M$. Let $V_{\sigma\left(j_{1}\right) j_{1}}=W_{\sigma\left(j_{1}\right) j_{1}}$ and let $V_{j_{1}, o\left(j_{1}\right)}=$ $\left(W_{\sigma\left(j_{1}\right) j_{1}}\right)^{*}$. Let $j_{2}$ be the next integer in $M$ such that $j_{2} \notin\left\{j_{1}, \sigma\left(j_{1}\right)\right\}$ and continue as before. Clearly $V$ is unitary and implements an automorphism of $\mathscr{D}(\mathcal{E})$. To verify that $V$ is selfadjoint, it suffices to show that for those $j \in M$ such that $\sigma(j)=j, V_{j j}=\left(V_{j j}\right)^{*}$. Since $U$ is selfadjoint so is $U_{j j}$, so that $W_{j j} P_{i j}=P_{j j}\left(W_{j j}\right)^{*}$ by definition and $W_{i j} P_{i j}=P_{j j} W_{i j}$ by [6, p. 69]. Since $P_{j j}$ is invertible (because $U_{j j}$ is), we conclude that $W_{j j}=\left(W_{j j}\right)^{*}$ and $V_{i j}=\left(V_{j j}\right)^{*}$ by construction. Finally, since $V E_{j} V^{*}=E_{\sigma(j)}$ for each $j$, we have that $\| U E_{j} U^{*}$ $-V E_{j} V^{*}\|=\| U E_{j} U^{*}-E_{\sigma(j)} \| \rightarrow 0$ following Lemma 7.

In the lemmas which follow, we adopt common usage and say that an operator $T \in \mathcal{L}(\mathcal{H})$ belongs to the essential commutant of a $C^{*}$-subalgebra $\mathbb{Q}$ of $\mathcal{L}(\mathcal{H C})$ if and only if $T S-S T \in \mathcal{C}(\mathcal{H})$ for every $S \in \mathbb{Q}$.

LEMMA 9. Suppose that $W$ is a unitary operator which implements an automorphism of $\mathbb{Q}=\mathscr{D}(\mathscr{E})+\mathcal{C}(\mathcal{H C})$ such that $\left\|W E_{j}-E_{j} W\right\| \rightarrow 0$. Then $W$ belongs to the essential commutant of the center of $\mathscr{D}(\mathcal{E})$.

Proof. It suffices to assume that $T$ belongs to the center of $\mathscr{D}(\mathcal{E})$ and then show that $T-W T W^{*}$ is compact. Since $W$ implements an automorphism of $\mathcal{Q}, W T W^{*}$ belongs to $\mathcal{Q}$. Hence, $\delta_{\varepsilon}\left[W T W^{*}\right]-W T W^{*}$ is compact by Proposition 4, and to prove the assertion, it suffices to show that $T-$ $\delta_{\mathscr{E}}\left[W T W^{*}\right]$ is compact; since both terms belong to $\mathscr{D}(\mathcal{E})$, this is equivalent to showing that $\left\|E_{j}\left[T-W T W^{*}\right] E_{j}\right\| \rightarrow 0$. Since $T$ belongs to the center of $\mathscr{D}(\mathscr{E}), T=\sum_{j=1}^{\infty} \lambda_{j} E_{j}$ for a bounded sequence of complex numbers $\left\{\lambda_{j}\right\}_{j=1}^{\infty}$; thus, 


$$
\begin{aligned}
&\left\|E_{j}\left[T-W T W^{*}\right] E_{j}\right\|=\left\|\lambda_{j} E_{j}-E_{j} W T W^{*} E_{j}\right\| \\
&<\left\|\lambda_{j} E_{j}-E_{j} W T E_{j} W^{*}\right\|+\left\|E_{j} W T\left(W^{*} E_{j}-E_{j} W^{*}\right)\right\| \\
&<\left\|\lambda_{j} E_{j}-W\left(\lambda_{j} E_{j}\right) W^{*}\right\|+\|T\| \cdot\left\|W^{*} E_{j}-E_{j} W^{*}\right\| \\
&<\left|\lambda_{j}\right| \cdot\left\|W^{*} E_{j}-E_{j} W^{*}\right\|+\|T\| \cdot\left\|W^{*} E_{j}-E_{j} W^{*}\right\| \rightarrow 0 .
\end{aligned}
$$

Hence, $W$ essentially commutes with every operator in the center of $\mathscr{D}(\mathcal{E})$.

LEMMA 2'. Let $U$ be a selfadjoint unitary operator which implements an automorphism of $\mathbb{Q}=\mathscr{D}(\mathscr{E})+\mathcal{C}(\mathcal{H})$. Then there is a selfadjoint unitary operator $V$ which implements an automorphism of $\mathscr{D}(\mathscr{E})$ such that $U^{*} V$ is a compact perturbation of the identity.

Proof. If $U$ is any selfadjoint unitary operator which implements an automorphism of $\mathscr{D}(\mathcal{E})+\mathcal{C}(\mathcal{H})$, then by Construction 8 we obtain an operator $V$ such that $U^{*} V$ implements an automorphism of $\mathscr{D}(\mathscr{E})+\mathcal{C}(\mathcal{H C})$ and $\left\|U^{*} V E_{j}-E_{j} U^{*} V\right\| \rightarrow 0$. Hence by Lemma $9, U^{*} V$ belongs to the essential commutant of the center of $\mathscr{D}(\mathcal{E})$; by an application of $[8$, Theorem 2.1] it follows that $U^{*} V$ belongs to [center $\left.(\mathscr{D}(\mathcal{E}))\right]^{\prime}+\mathcal{C}(\mathcal{H})$. Consequently, $U^{*} V$ belongs to $\mathscr{D}(\mathscr{E})+\mathcal{C}(\mathcal{H C})$. Now

$$
\begin{aligned}
1-U^{*} V & =\delta_{\mathscr{E}}\left(1-U^{*} V\right)+\left[1-U^{*} V-\delta_{\mathscr{E}}\left(1-U^{*} V\right)\right] \\
& =\delta_{\mathscr{E}}\left(1-U^{*} V\right)-\left[U^{*} V-\delta_{\mathscr{\delta}}\left(U^{*} V\right)\right],
\end{aligned}
$$

where $U^{*} V-\delta_{\S}\left(U^{*} V\right)$ is compact by Proposition 4. So, to prove the assertion, it suffices to show that $\delta_{\mathscr{E}}\left(1-U^{*} V\right)$ is compact. But, that follows from a matrix multiplication: if $j \in M$, then

$$
\begin{aligned}
\left\|E_{j}\left(1-U^{*} V\right) E_{j}\right\| & =\left\|E_{j}-E_{j}\left(U^{*}\right)_{j, \sigma(j)} V_{\sigma(j) j} E_{j}\right\| \\
& =\left\|E_{j}-E_{j}\left(U_{\sigma(j) j}\right)^{*} V_{\sigma(j) j} E_{j}\right\| \\
& =\left\|E_{j}-E_{j}\left(P_{\sigma(j) j}\right)\left(W_{\sigma(j) j}\right)^{*} W_{\sigma(j) j} E_{j}\right\| \\
& =\left\|E_{j}-E_{j}\left(P_{\sigma(j) j}\right) E_{j}\right\| \\
& =\left\|E_{j}\left[1_{E, \mathcal{C}}-\left(P_{\sigma(j) j}\right)\right] E_{j}\right\| \\
& \leqslant\left\|1_{E, \mathcal{X}}-P_{\sigma(j) j}\right\| .
\end{aligned}
$$

We remark that $P_{\sigma(\rho) j}$ is a positive invertible operator on the finite dimensional space $E_{j} \mathcal{H}$. So for $j \in M$ 


$$
\begin{aligned}
& \left\|1_{E, X}-P_{\sigma(j), j}\right\|=1-\inf \left\{\mu: \mu \in \operatorname{spectrum}\left(P_{\sigma(j) j}\right)\right\} \\
& =1-\inf _{x \in E_{j} j \mathcal{C}}\left\|P_{\sigma(j) j} x\right\| \\
& \|x\|=1 \\
& =1-\inf _{\substack{x \in E_{j} \mathcal{S C} \\
\|x\|=1}}\left\|W_{\sigma(j)_{j} j} P_{\sigma(j), j} x\right\| \quad \text { since } W_{\sigma(j), j} \text { is unitary } \\
& \leqslant 1-\inf _{x \in E_{j} \mathcal{X C}}\left\|E_{o(j)} U E_{j} x\right\|^{2} \rightarrow 0 \\
& \|x\|=1 \\
& \text { since } \inf _{\substack{x \in E_{j} \mathcal{X} \\
\|x\|=1}}\left\|E_{\sigma(j)} U E_{j} x\right\|^{2}=1-\left\|E_{\sigma(j)}^{\perp} U E_{j}\right\|^{2} \text {. }
\end{aligned}
$$

Because $\mathbf{N}-M$ is finite, it follows that $\left\|E_{j}\left(1-U^{*} V\right) E_{j}\right\| \rightarrow 0$, or equivalently, that $\delta_{\S}\left(1-U^{*} V\right)$ is compact, from which we conclude that $1-U^{*} V$ is compact.

REMARK 10. Suppose that $W$ is a unitary operator which implements an automorphism of $\mathscr{D}(\mathcal{E})+C(\mathcal{C})$. Then $W$ is a compact perturbation of a partial isometry $Y$ of index zero such that $\left\{Y E_{i} Y^{*}\right\}_{i=1}^{\infty} \subset \mathcal{E} \cup\{0\}$.

Proof. Adopting the notation of Theorem 1 , we let $\mathscr{D}(\mathscr{F})=\mathscr{D}(\mathcal{E})$ and $P$ be the projection onto the first coordinate space of $\mathscr{K}=\mathcal{H} \oplus \mathcal{H}$. So, $W=\left.P^{\perp} U\right|_{P K}=\left.P^{\perp} V\right|_{P \mathcal{K}}-\left.P^{\perp} Q\right|_{P \mathscr{K}}$; let $Y=\left.P^{\perp} V\right|_{P \mathcal{K}}$ and the assertion is easily verified after noting that $\operatorname{ker}(Y)=\left(\Sigma_{i \in N_{0}} E_{i}\right) \mathcal{H}$ and $\operatorname{ker}\left(Y^{*}\right)=$ $\left(\Sigma_{j \in N_{1}} E_{j}\right) \mathcal{H}$.

DEFINITION. Let $W$ be a unitary operator which implements an automorphism of $\mathcal{Q}+\mathcal{C}(\mathcal{H})$, where $\mathbb{Q}$ is a $C^{*}$-algebra. We say that $W$ splits (with respect to $Q)$ if it is a compact perturbation of a unitary operator which implements an automorphism of $\mathbb{Q}$.

It should be mentioned that Lemma 2 is not a surprising result as it is suggested by [ 8 , Theorem 4.10] which asserts that every unitary which implements an automorphism of $\mathbb{Q}+\mathcal{C}(\mathcal{H})$ splits whenever $\mathbb{Q}$ is a maximal abelian selfadjoint subalgebra of $\mathcal{L}(\mathcal{C})$. However, our situation differs in that there are unitary operators which implement automorphisms of perturbed block diagonal algebras which do not split.

In the following corollaries, $P_{k}$ will denote $\Sigma\left\{E_{i}: \operatorname{dim} E_{i}=k\right\}$.

COROLLARY 11. Let $U$ be a unitary operator which implements an automorphism of $\mathscr{D}(\mathcal{E})+\mathcal{C}(\mathcal{H})$. $U$ splits if and only if

$$
\text { index }\left(P_{k} U_{P_{k} \Im}\right)=0 \text { for every } k \in \mathbf{N} \text {. }
$$

PROOF. $\Rightarrow$ : By assumption there exists a unitary operator $V$ which commutes with $P_{k}$ for each $k$ and a compact $C$ such that $U=V+C$. Since index is invariant under compact perturbation [4], 


$$
\text { index }\left(\left.P_{k} U\right|_{P_{k} \Im C}\right)=\operatorname{index}\left(\left.P_{k} V\right|_{P_{k} \Im}\right)=0 .
$$

$\Leftarrow$ : From Remark 10, $U=W+C$, where $C$ is compact and $W$ is a partial isometry of index zero such that $\left\{W E_{i} W^{*}\right\}_{i=1}^{\infty} \subset \mathcal{E} \cup\{0\}$. Note that $\left.P_{k} W\right|_{P_{k}}$ iC is unitary for all $k \notin R$, where $R$ is a finite subset of $\mathbf{N}$; for each $k \in R$, index $\left(\left.P_{k} W\right|_{P_{k}}\right.$ s $)=0$, so

$$
\begin{aligned}
\operatorname{ker}\left(\left.P_{k} W\right|_{P_{k} \Im \mathcal{C}}\right) & =\sum_{i \in N_{k}} E_{i} \text { and } \\
\operatorname{cokernel}\left(\left.P_{k} W\right|_{P_{k} \Im}\right) & =\sum_{i \in 0_{k}} E_{i} \quad \text { with } \operatorname{card}\left(N_{k}\right)=\operatorname{card}\left(0_{k}\right)<\infty .
\end{aligned}
$$

Define $F_{k}$, a finite rank partial isometry such that $\left\{F_{k} E_{i} F_{k}^{*}\right\}_{i \in N_{k}}=\left\{E_{i}\right\}_{i \in 0_{k}}$, and note that $U$ is a compact perturbation of a unitary operator $W+$ $\Sigma_{k \in R} F_{k}$ which implements an automorphism of $\mathscr{D}(\mathcal{E})$.

In Corollary 11 we presented a necessary and sufficient condition for a specific unitary operator to split. In Corollary 12 we obtain a necessary and sufficient condition for a perturbed block diagonal algebra to have the property that every unitary implementing an automorphism of it splits:

COROLLARY 12. Every unitary operator which implements an automorphism of $\mathscr{D}(\mathcal{E})+C(\mathcal{H})$ splits if and only if at most one projection $P_{k}$ is infinite dimensional.

Proof. $\Rightarrow$ : We assume that $P_{k}$ and $P_{l}$ are infinite dimensional for $k \neq l$ and construct a unitary $U$ that implements an automorphism of $\mathscr{D}(\mathscr{E})+$ $\mathcal{C}(\mathcal{H})$ but does not split. For each $j$, let $\operatorname{support}\left(P_{j}\right)=\left\{i: \operatorname{dim} E_{i}=j\right\}$ and let $\left\{M_{i}\right\}_{i=1}^{\infty}$ and $\left\{N_{i}\right\}_{i=1}^{\infty}$ be partitions of $\operatorname{support}\left(P_{k}\right)$ and $\operatorname{support}\left(P_{l}\right)$ respectively such that card $M_{i}=l$ and card $N_{i}=k$ for all $i$. Let $U$ be any unitary operator such that $U E_{i} U^{*}=E_{i}$ for $i \notin \operatorname{support}\left(P_{k}\right) \cup \operatorname{support}\left(P_{l}\right)$ and

$$
\begin{aligned}
U\left(\sum_{i \in M_{1}} E_{i}\right) U^{*} & =\sum_{i \in N_{1}} E_{i}, \\
\left\{U E_{i} U^{*}\right\}_{i \in M_{k}} & =\left\{E_{i}\right\}_{i \in M_{k-1},}, k>1, \\
\left\{U E_{i} U^{*}\right\}_{i \in N_{k}} & =\left\{E_{i}\right\}_{i \in N_{k+1}}, \quad k>1,
\end{aligned}
$$

and note that $U$ implements an automorphism of $\mathscr{D}(\mathcal{E})+\mathcal{C}(\mathcal{H})$, but that index $\left(\left.P_{k} U\right|_{P_{k} \text { s }}\right)=k l \neq 0$, so that by Corollary $11, U$ does not split.

$\Leftarrow$ : This is a routine argument modulo Remark 10 .

We recall that an automorphism of an algebra is said to be inner if it can be implemented by a unitary which belongs to the algebra.

COROLlaRY 13. All automorphisms of $\mathbb{Q}=\mathcal{D}(\mathcal{E})+\mathcal{C}(\mathcal{H})$ are inner if and only if there exists a positive integer $l$ such that whenever $n, m>l, n \neq m$, then $\operatorname{dim}\left(E_{n}\right) \neq \operatorname{dim}\left(E_{m}\right)$. 
Proof. $\Leftarrow$ : Every automorphism $\alpha$ of $\mathscr{Q}$ is implemented by a unitary operator $U_{\alpha}$ (by a slight variation of [8, Lemma 4.5]). By Remark 10, $U_{\alpha}=W+C$, where $C$ is compact and $W$ is a partial isometry of index zero such that $\left\{W E_{i} W^{*}\right\}_{i=1}^{\infty} \subseteq \mathcal{E} \cup\{0\}$ so it follows that $U$ belongs to $\mathbb{Q}$ iff $W$ does. Since $W$ must map the $E_{i}$ to other $E_{i}$ of the same dimension, the assumption implies that $W E_{i} W^{*}=E_{i}$ for all but finitely many $i>l$ and that $W$ belongs to $\mathbb{Q}$.

$\Rightarrow$ : In this proof by the contrapositive, we assume that for every positive integer $l$ there exist $n, m>l, n \neq m$, such that $\operatorname{dim} E_{n}=\operatorname{dim} E_{m}$ and then assert that there is a unitary operator $U$ which implements an automorphism of $\mathscr{D}(\mathcal{E})$ (hence, $\mathscr{D}(\mathcal{E})+\mathcal{C}(\mathcal{H})$ ) but which does not belong to $\mathcal{Q}$. We leave this construction to the reader and then remark that the automorphism $\alpha$ which is induced by $U$ cannot be implemented by any unitary operator which belongs to $\mathbb{Q}$ and therefore is not inner.

Corollary 14. Suppose $\mathbb{Q}=\mathscr{D}(\mathscr{E})+\mathcal{C}(\mathcal{F C})$ and $\mathscr{B}=\mathscr{D}(\mathscr{F})+\mathcal{C}(\mathcal{F C})$. The following are equivalent:

(i) $\mathbb{Q}=\mathscr{B}$.

(ii) There exist finite subsets $O_{1}$ and $O_{2}$ of $\mathrm{N}, a$ unitary operator $U$ which is a compact perturbation of the identity, and a bijection $\sigma: \mathbf{N}-O_{1} \rightarrow \mathbf{N}-O_{2}$ such that $U\left(\Sigma_{i \in O_{1}} E_{i}\right) U^{*}=\Sigma_{j \in O_{2}} F_{j}$ and $U E_{i} U^{*}=F_{o(i)}$ for $i \in \mathbf{N}-O_{1}$.

(iii) There exist finite subsets $O_{1}$ and $O_{2}$ of $\mathbf{N}$ and a bijection $\sigma: N-O_{1} \rightarrow \mathbf{N}$ $-\mathrm{O}_{2}$ which satisfies this condition: for every $\varepsilon>0$ there is a finite subset $N(\varepsilon)$ of $\mathbf{N}$ such that

$$
\left\|\sum_{i \in N(\varepsilon) \cup O_{1}} \lambda_{i} E_{i}-\sum_{i \in N(\varepsilon) \cup O_{1}} \lambda_{i} F_{o(i)}\right\|<\varepsilon
$$

for every sequence $\left\{\lambda_{i}\right\}_{i \in N(\varepsilon) \cup O_{1}}$ of zeros and ones.

Proof. (i) $\Rightarrow$ (ii): $\mathscr{Q}=\mathscr{B}$ implies that $\mathscr{Q} \simeq \mathscr{B}$ and by Theorem 1 the existence of a unitary operator $V$ such that for finite sets $N_{1}$ and $N_{2}$ and a bijection $\gamma: \mathrm{N}-N_{1} \rightarrow \mathrm{N}-N_{2}, V\left(\Sigma_{i \in N_{1}} E_{i}\right) V^{*}=\Sigma_{j \in N_{2}} F_{j}$ and $V E_{i} V^{*}=F_{\gamma(i)}$ for all $i \notin N_{1}$. Since $V$ implements an automorphism of $\mathbb{Q}, V=W+Q$, where $Q \in \mathcal{C}(\mathcal{F C})$ and $W$ is a unitary operator for which there exist finite subsets $M_{1}$ and $M_{2}$ of $\mathrm{N}$ and a bijection $\tau: \mathrm{N}-M_{1} \rightarrow \mathrm{N}-M_{2}$ such that $W\left(\Sigma_{i \in M_{1}} E_{i}\right) W^{*}=\Sigma_{i \in M_{2}} E_{i}$ and $W E_{i} W^{*}=E_{\tau(i)}$ for all $i \notin M_{1}$. Hence $V W^{*}$ $=1+Q W^{*}$ and the reader may verify that if $O_{1}=M_{2} \cup \tau\left(N_{1}-N_{1} \cap M_{1}\right)$ and $O_{2}=N_{2} \cup \gamma\left(M_{1}-M_{1} \cap N_{1}\right)$ then $\sigma=\gamma \circ \tau^{-1}$ is a bijection from $\mathbf{N}-O_{1}$ to $\mathbf{N}-O_{2}$ such that if $U=V W^{*}, U\left(\Sigma_{i \in O_{1}} E_{i}\right) U^{*}=\Sigma_{j \in O_{2}} F_{j}$ and $U E_{i} U^{*}=F_{\sigma(i)}$ for all $i \notin O_{1}$.

(ii) $\Rightarrow$ (iii): Since $U=1+K$ for $K$ compact, 


$$
\begin{aligned}
\sum_{i \in O_{1}} \lambda_{i} F_{\mathrm{o}(i)}= & U\left(\sum_{i \in O_{1}} \lambda_{i} E_{i}\right) U^{*}=\sum_{i \in O_{1}} \lambda_{i} E_{i}+K\left(\sum_{i \in O_{1}} \lambda_{i} E_{i}\right) \\
& +\left(\sum_{i \in O_{1}} \lambda_{i} E_{i}\right) K^{*}+K\left(\sum_{i \in O_{1}} \lambda_{i} E_{i}\right) K^{*}
\end{aligned}
$$

and

$$
\left\|\sum_{i \in O_{1}} \lambda_{i} F_{\mathrm{o}(i)}-\sum_{i \in O_{1}} \lambda_{i} E_{i}\right\| \leqslant 3 \max \{1,\|K\|\} \cdot\left\|K\left(\sum_{i \in O_{1}} \lambda_{i} E_{i}\right)\right\|
$$

for every sequence $\left\{\lambda_{i}\right\}$ of zeros and ones. Because $K$ is compact, given $\varepsilon>0$, there exists a subset $N(\varepsilon)$ such that

$$
\left\|K\left(\sum_{i \in N(\varepsilon) \cup O_{1}} \lambda_{i} E_{i}\right)\right\| \leqslant \frac{\varepsilon}{3 \max \{1,\|K\|\}}
$$

for every sequence of zeros and ones.

(iii) $\Rightarrow$ (i): We assert that $\sum_{i \notin O_{1}} \lambda_{i} E_{i}-\sum_{i \in O_{1}} \lambda_{i} F_{o(i)}$ is compact for every sequence $\left\{\lambda_{i}\right\}_{i \in O_{1}}$ of zeros and ones; for, let

$$
P_{k}=\left(\sum_{i \in O_{1}} E_{i}\right) \vee\left(\sum_{j \in O_{2}} F_{j}\right) \vee E_{i_{1}} \vee F_{\sigma\left(i_{1}\right)} \cdots \vee E_{i_{k}} \vee F_{\sigma\left(i_{k}\right)},
$$

where $i_{k}$ is the $k$ th integer in $\mathbf{N}-O_{1}$, and note that $P_{k}^{\perp} \downarrow 0$, and that

$$
\left\|\left(\sum_{i \in O_{1}} \lambda_{i} E_{i}-\sum_{i \in O_{1}} \lambda_{i} F_{\sigma(i)}\right) P_{k}^{\perp}\right\|<\left\|\sum_{\substack{i \in O_{1} \\ i>k}} \lambda_{i} E_{i}-\sum_{\substack{i \in O_{1} \\ i>k}} \lambda_{i} F_{\sigma(i)}\right\| \rightarrow 0
$$

by (iii). It is not difficult to show that the essential center of $\mathbb{Q}$ is generated by $\left\{\sum_{i=1}^{\infty} \lambda_{i} E_{i}: \lambda_{i}=0\right.$ or 1$\}+\mathcal{C}(\mathcal{H C})$ and to conclude that the essential center of $\mathbb{Q}$ is equal to the essential center of $\mathscr{B}$ so that by [8, Theorem 2.1$], \mathbb{Q}=\mathscr{B}$.

Since $\mathcal{C}(\mathcal{K})$ is a norm-closed, two sided *-ideal in $\mathcal{L}(\mathcal{H})$, the quotient $\mathcal{L}(\mathcal{H}) / \mathcal{C}(\mathcal{H C})$ is a $C^{*}$-algebra commonly referred to as the Calkin algebra and $\pi: \mathcal{L}(\mathcal{H}) \rightarrow \mathcal{L}(\mathcal{H}) / \mathcal{C}(\mathcal{H})$ is the usual projection map, taking $T$ to its coset $\{T+K: K \in \mathcal{C}(\mathcal{H})\}$.

Definition. Suppose that $\mathcal{Q}$ and $\mathscr{B}$ are $C^{*}$-subalgebras of the Calkin algebra. $\mathcal{Q}$ and $\mathscr{B}$ are said to be essentially unitarily equivalent if and only if some unitary operator in the Calkin algebra implements an isomorphism of them.

Corollary 15. Let $\mathbb{Q}=\mathscr{D}(\mathcal{E})+\mathcal{C}(\mathcal{H})$ and $\mathscr{B}=\mathscr{D}(\mathscr{F})+\mathcal{C}(\mathcal{H}) \cdot \pi(\mathbb{Q})$ and $\pi(\mathscr{B})$ are essentially unitarily equivalent if and only if there exist finite sets 
$N_{0}$ and $N_{1}$ and a bijection $\tau: \mathbf{N}-N_{0} \rightarrow \mathbf{N}-N_{1}$ such that $\operatorname{dim}\left(E_{i}\right)=\operatorname{dim}\left(F_{\tau(i)}\right)$ for every $i \in \mathbf{N}-N_{0}$.

Proof. $\Leftarrow$ : Let $W$ be a partial isometry with $\operatorname{ker} W=\Sigma_{i \in N_{0}} E_{i}$ and coker $W=\Sigma_{j \in N_{1}} F_{j}$ such that $W E_{i} W^{*}=F_{\tau(i)}$ for all $i \in \mathbf{N}-N_{0}$. Clearly, $\pi(W)$ is unitary and $\pi(W) \pi(\mathbb{Q}) \pi(W)^{*}=\pi(\mathscr{B})$.

$\Rightarrow$ : Assuming that $\pi(W)$ implements an isomorphism of $\pi(\mathscr{Q})$ and $\pi(\mathscr{B})$, then $U=\left(\begin{array}{c}W \\ W\end{array} W^{* *}\right)$ is selfadjoint and $\pi(U)$ is unitary; one can verify that $U$ is a compact perturbation of a selfadjoint unitary $R$ and that this selfadjoint unitary implements an automorphism of $\mathscr{D}(\mathscr{E}) \oplus \mathscr{D}(\mathscr{F})+\mathcal{C}(\mathcal{H} \oplus \mathscr{H})$. From Lemma 2, $R$ is a compact perturbation of a selfadjoint unitary $V$ which implements an automorphism of $\mathscr{D}(\mathcal{E}) \oplus \mathscr{D}(\mathscr{F})$. Now adopting the notation and arguing as in the proof of Theorem 1, since $\tau$ is dimension-preserving it suffices to observe that $N_{0}$ and $N_{1}$ are finite subsets.

We now shift our attention to a sequence $\mathcal{P}=\left\{P_{n}\right\}_{n=1}^{\infty}$ of finite dimensional projections increasing to the identity and to the algebras which are naturally associated with it. The triangular algebra associated with $\mathcal{P}$ and denoted as $\mathscr{T}(\mathcal{P})$ is defined to be the set of those operators which leave all of the projections of $\mathscr{P}$ invariant. The quasitriangular algebra associated with $\mathcal{P}$ and denoted as $2 \mathcal{T}(\mathcal{P})$ is defined to be the set of operators $T$ for which $\left\|P_{n}{ }^{\perp} T P_{n}\right\| \rightarrow 0$. It is easy to verify that $\mathscr{T}(\mathcal{P})+\mathcal{C}(\mathcal{F C})$ is contained in $2 \mathcal{T}(\mathcal{P})$; W. Arveson proved, in fact, that $\mathscr{T}(\mathcal{P})+\mathcal{C}(\mathcal{H C})=2 \mathcal{T}(\mathcal{P})[1]$.

Definition. An algebra $Q$ is called quasidiagonal for a sequence $\mathcal{P}$, and denoted as $\mathscr{2} \mathscr{D}(\mathscr{P})$, if it consists of those operators $T$ for which $\| T P_{n}-$ $P_{n} T \| \rightarrow 0$.

If $\mathscr{D}(\mathcal{P})$ denotes the set of those operators which commute with the elements of $\mathscr{P}$, then it is easy to see that $\mathscr{D}(\mathscr{P})+\mathcal{C}(\mathcal{H})$ is simply a perturbed block diagonal algebra and is contained in $\mathscr{Q} \mathscr{D}(\mathscr{P})$. It was already known that for every $T$ in $\mathcal{Q} \mathscr{D}(\mathcal{P})$ there is a subsequence $\left\{P_{n_{k}}\right\}_{k=1}^{\infty}$ of $\mathcal{P}$, dependent on $T$, for which $T$ belongs to $\mathscr{D}\left(\left\{P_{n_{k}}\right\}_{k=1}^{\infty}\right)+\mathcal{C}(\mathcal{H C})$ [7]. The natural question, suggested by the nonselfadjoint case, is whether $\mathscr{D}(\mathcal{P})+\mathcal{C}(\mathcal{F})$ equals $2 \mathscr{D}(\mathcal{P})$. In Lemma 17 we show that it does not.

Proposition 16. $2 \mathscr{D}(\mathcal{P})=2 \mathcal{T}(\mathscr{P}) \cap 2 \mathcal{T}(\mathcal{P})^{*}$.

Proof. $\subseteq:$ If $T \in 2 \mathscr{D}(\mathcal{P})$ then $\left\|T P_{n}-P_{n} T\right\| \rightarrow 0$ so $\left\|P_{n}^{\perp} T P_{n}\right\|=$ $\left\|P_{n}^{\perp}\left(T P_{n}-P_{n} T\right)\right\| \rightarrow 0$; by applying the same argument to the adjoint of $T P_{n}-P_{n} T$, we have that $\left\|P_{n}^{\perp} T^{*} P_{n}\right\|=\left\|P_{n} T P_{n}{ }^{\perp}\right\| \rightarrow 0$. Hence, $T \in \mathcal{Q} \mathcal{S}(\mathcal{P})$ $\cap 2 \mathcal{T}(\mathscr{P})^{*}$.

卫: If $T \in \mathcal{2} \mathcal{T}(\mathcal{P}) \cap 2 \mathcal{T}(\mathcal{P})^{*}$ then $\left\|T P_{n}-P_{n} T\right\| \leqslant\left\|\left(1-P_{n}\right) T P_{n}\right\|+$ $\left\|P_{n} T\left(1-P_{n}\right)\right\| \rightarrow 0$, so $T \in \mathcal{Q} \mathscr{D}(\mathcal{P})$.

DEFinition. Let $\mathcal{T C}$ be a Hilbert space and $M$ be the matrix of an operator with respect to a fixed orthonormal basis $\left\{e_{i}\right\}$. Define ${ }_{x}: M \rightarrow M^{+}$to be 
the linear map on matrices which takes $M$ to a matrix derived from it by replacing all entries below the main diagonal with zeros. Such maps have been studied before [5], [10].

$$
\text { LEMMA 17. } \mathscr{D}(\mathscr{P})+\mathcal{C}(\mathcal{H}) \underset{\neq}{\mathcal{Q}} \mathscr{D}(\mathscr{P}) \text {. }
$$

Proof. To simplify the notation, we assume first that $\operatorname{dim} P_{n}=n$ for all $n$. Let $\left\{e_{k}\right\}_{k=1}^{\infty}$ be an orthonormal set in $\mathcal{H}$ such that $e_{n} \in\left(P_{n} \ominus P_{n-1}\right) \mathcal{H}$ for each $n$. Let $\mathcal{H}_{i}=\operatorname{span}\left\{e_{k}: i^{2} \leqslant k<(i+1)^{2}\right\}$ and for each $i$ let $C_{i} \in \mathcal{L}\left(\mathcal{H}_{i}\right)$ with $\left\|C_{i}^{+}\right\|=1$ and $\left\|C_{i}\right\|=\left\|+{ }_{i}\right\|^{-1}$. Put $C=C_{1} \oplus C_{2} \oplus \cdots$ so that $C^{+}$ $=C_{1}^{+} \oplus C_{2}^{+} \oplus \cdots$ is not compact; however, $\operatorname{dim} \mathcal{K}_{i} \rightarrow \infty$ so that $\left\|+_{i}\right\|$ $\rightarrow \infty\left[9\right.$, Proposition 1.2] and $C$ is compact. Hence $\delta\left(C^{+}\right)=\delta(C)$ is compact so $C^{+}-\delta\left(C^{+}\right)$is not compact and $C^{+} \notin \mathscr{D}(\mathscr{P})+\mathcal{C}(\mathcal{H C})$. However, $C^{+} \in$ $\mathcal{T}(\mathcal{P})$ and $C^{+}=C-\left(C-C^{+}\right) \in \mathcal{T}(\mathcal{P})^{*}+\mathcal{C}(\mathcal{H})$ because $C-C^{+} \in$ $\mathscr{T}(\mathscr{P})^{*}$; hence, $C^{+} \in \mathcal{Q} \mathscr{Q}(\mathscr{P})$ by Proposition 16 . To generalize the argument to an arbitrary sequence $\mathcal{P}$, construct $C_{i}$ as before and define $D_{i} \in$ $\mathcal{L}\left(P_{(i+1)^{2}} \mathcal{H} \Theta P_{i^{2}} \mathcal{H}\right)$ by $D_{i}=C_{i} \oplus 0$ so that $D=D_{1} \oplus D_{2} \oplus \cdots$ is compact and $D^{+}$belongs to $2 \mathscr{D}(\mathscr{P})$ but not to $\mathscr{D}(\mathcal{P})+\mathcal{C}(\mathcal{H})$.

The interested reader can verify that $\mathscr{D}(\mathscr{P})+\mathcal{C}(\mathcal{H})$ is equal to the norm closure of $[\mathcal{T}(\mathscr{P})+\mathscr{G}] \cap\left[\mathcal{F}(\mathcal{P})^{*}+\mathscr{G}\right]$, where $\mathcal{G}$ is the ideal of HilbertSchmidt operators. We remark that Lemma 17 together with Proposition 16 and the fact that $2 \mathcal{T}(\mathscr{P})=\mathscr{T}(\mathscr{P})+\mathcal{C}(\mathcal{H})[1]$ says that $\mathscr{D}(\mathscr{P})+\mathcal{C}(\mathcal{H}) \subsetneq$ $[\mathcal{T}(\mathscr{P})+\mathcal{C}(\mathcal{H})] \cap\left[\mathcal{T}(P)^{*}+\mathcal{C}(\mathcal{H})\right]$. Nevertheless, we can obtain $\mathscr{D}(\mathcal{P}) \neq$ $\mathcal{C}(\mathcal{H})$ as the intersection of certain quasidiagonal algebras associated with the sequence $\mathcal{P}$. To do so, let $\pi$ be any permutation of the positive integers and let $E_{n}=P_{n} \ominus P_{n-1}$ for each positive $n$; then $P_{n}^{\pi}$ will denote $\Sigma\left\{E_{i}: \pi(i)<n\right\}$ and $\mathcal{Q}^{\pi}(\mathscr{P})$ will denote the quasidiagonal algebra of those operators $T$ for which $\left\|P_{n}^{\pi} T-T P_{n}^{\pi}\right\| \rightarrow 0$.

THEOREM 18. $\mathscr{D}(\mathscr{P})+\mathcal{C}(\mathcal{H})=\bigcap_{\pi} \mathscr{Q}^{\pi}(\mathscr{P})$, where we intersect over all permutations $\pi$.

Proof. Clearly $\subseteq$ holds. To verify $\supseteq$, it suffices to assume $T \notin \mathscr{D}(\mathscr{P})+$ $\mathcal{C}(\mathcal{H})$ and then show that $T \notin \cap_{\pi} \mathcal{Q}^{\pi}(\mathscr{P})$. By [8, Theorem 2.1] we know that there exists an operator $Q \in \mathscr{D}(\mathscr{P})^{\prime}$ such that $Q T-T Q \notin \mathcal{C}(\mathcal{H})$; since every operator in $\mathscr{D}(\mathscr{P})^{\prime}$ can be approximated in norm by finite linear combinations of projections in $\mathscr{Q}(\mathcal{P})^{\prime}$, we can assume that $Q$ is a projection. Hence, either $Q T Q^{\perp}$ or $Q^{\perp} T Q$ fails to be compact and without loss of generality we assume the former. So, there exist $\varepsilon>0$ and a sequence of finite dimensional, mutually orthogonal projections $\left\{F_{i}, G_{i}\right\}_{i=1}^{\infty} \subset \mathscr{D}(\mathcal{P})^{\prime}$ such that $F_{i}=\Sigma_{k \in M_{i}} E_{k}<Q$ and $G_{i}=\Sigma_{k \in N_{i}} E_{k}<Q^{\perp}$ for all $i$ and $\inf _{i}\left\|F_{i} T G_{i}\right\| \geqslant \varepsilon$. Now we define a permutation $\pi$ of $\mathbf{N}$ for which $T \notin$ $\mathcal{Q} \mathscr{D}^{\pi}(\mathscr{P})$. Let $\pi \operatorname{map} N_{1}$ to the first card $\left(N_{1}\right)$ integers and map $M_{1}$ to the next 
$\operatorname{card}\left(M_{1}\right)$ integers. Let $R_{1}=\left\{j \in \mathrm{N}: j \notin \cup_{i \in \mathrm{N}}\left(M_{i} \cup N_{i}\right)\right.$ and $j<\operatorname{card}\left(N_{1}\right)$ $\left.+\operatorname{card}\left(M_{1}\right)\right\}$. Map $R_{1}$ to the next $\operatorname{card}\left(R_{1}\right)$ integers. Continue inductively with

$$
\begin{aligned}
R_{k+1}=\left\{J \in \mathrm{N}: j \notin\left(\bigcup_{i \in \mathrm{N}}\left(M_{i} \cup N_{i}\right)\right) \cup_{i<k} R_{i}\right. \\
\left.\quad \text { and } j<\sum_{i<k+1}\left(\operatorname{card}\left(M_{i}\right)+\operatorname{card}\left(N_{i}\right)\right)\right\}
\end{aligned}
$$

and note that $\pi$ is a bijection of $\mathbf{N}$ and that $T \notin \mathscr{Q} \mathscr{Q}^{\pi}(\mathscr{P})$.

If $\mathcal{S}$ is a subset of $\mathcal{L}(\mathcal{H})$, then we define $\operatorname{lat}_{f}(\mathcal{S})$ to be the set of finite dimensional projections $P$ on $\mathcal{C}$ such that $P^{\perp} T P=0$ for every $T$ in $\delta$. The reader can easily verify that if $\mathscr{E}=\left\{E_{n}\right\}_{n=1}^{\infty}$ is a sequence of mutually orthogonal, finite dimensional projections on $\mathcal{H}$ whose sum is the identity, that $R$ belongs to lat $\mathcal{D}_{f}(\mathscr{E})$ if and only if $R$ is a finite sum of $E_{n}$ 's. We view lat ${ }_{f} \mathcal{D}(\mathcal{E})$, ordered by range inclusion, as a directed set and $R \rightarrow\left\|R^{\perp} T R\right\|$ as a net on that directed set. We offer this intrinsic characterization of a perturbed block diagonal algebra based on Theorem 18:

Corollary 19.

$$
T \in \mathscr{D}(\mathcal{E})+\mathcal{C}(\mathcal{H}) \quad \text { iff } \quad \lim _{R \rightarrow 1}\left\|R^{\perp} T R\right\|=0 .
$$

We remark that $\lim _{R \rightarrow 1} \sup \left\|R^{\perp} T R\right\|=\varepsilon>0$ means that for every $\eta<\varepsilon$ and every $R_{0} \in \operatorname{lat}_{f} \mathscr{D}(\mathscr{E})$ there exists $R \in$ lat $_{f} \mathscr{D}(\mathscr{E})$ such that $R>R_{0}$ and $\left\|R^{\perp} T R\right\|>\eta$.

Proof. $\Rightarrow$ : Immediate.

$\Leftarrow$ : We assume that $T \dot{\notin} \mathscr{D}(\mathscr{E})+\mathcal{C}(\mathcal{H})$ and show that $\lim _{R \rightarrow 1}$ $\sup \left\|R^{\perp} T R\right\|>0$. Note that the construction of Theorem 18 actually proves that $\mathscr{D}(\mathscr{E})+\mathcal{C}(\mathcal{H C})$ is the intersection of all quasitriangular algebras $2 \mathcal{T}\left(\left\{\Sigma_{\pi(i)<n} E_{i}\right\}_{n=1}^{\infty}\right)$, where $\pi$ is any permutation of $\mathbf{N}$. Hence, if $T \notin \mathscr{D}(\mathscr{E})$ $+\mathcal{C}(\mathcal{H C})$, then there is a permutation $\pi$ of $\mathbf{N}$ and $\varepsilon>0$ such that $\left\|\left(\Sigma_{\pi(i)<n} E_{i}\right)^{\perp} T\left(\Sigma_{\pi(i)<n} E_{i}\right)\right\|=\varepsilon$, and the reader can easily verify that $\lim _{R>1}$ $\sup \left\|R^{\perp} T R\right\|=\varepsilon$, thus concluding the argument.

THEOREM 20. The essential commutant of $\mathcal{2} \mathscr{D}(\mathcal{P})$ is $\mathbf{C} \cdot 1+\mathcal{C}(\mathcal{H})$.

Proof. To simplify the notation we again assume that $\operatorname{dim} P_{n}=n$ for all $n$ and remark that the most general case can be argued from this. Since $\mathscr{D}(\mathscr{P})+\mathcal{C}(\mathcal{H}) \subseteq \mathcal{L} \mathscr{D}(\mathcal{P})$, it follows that the essential commutant of $\mathscr{D}(\mathscr{P})$ is contained in the essential commutant of $\mathscr{D}(\mathcal{P})+\mathcal{C}(\mathcal{H})$ which by $\left[8\right.$, Theorem 2.1] is $\mathscr{D}(\mathcal{P})^{\prime}+\mathcal{C}(\mathcal{H})$. If the essential commutant of $\mathscr{Q} \mathscr{D}(\mathscr{P})$ is larger than $\mathbf{C} \cdot 1+\mathcal{C}(\mathcal{H})$, then it must contain a unitary operator $U$ whose 
Weyl spectrum has at least two distinct points $\lambda_{1}$ and $\lambda_{2}$. So, $U=V+K$, where $K$ is compact and $V \in \mathscr{D}(\mathscr{P})^{\prime}$ and by perturbing by another compact operator if necessary we insure that $V=\sum_{i=1}^{\infty} \mu_{i} E_{i}$, for $E_{i}=P_{i} \ominus P_{i-1}$, with $\lambda_{1}$ and $\lambda_{2}$ repeated infinitely of ten in the sequence $\left\{\mu_{i}\right\}_{i=1}^{\infty}$. Therefore, there is a subsequence $\left\{i_{k}\right\}_{k=1}^{\infty}$ of $N$ such that

$$
V=\sum_{k \text { even }} \lambda_{1} E_{i_{k}} \oplus \sum_{k \text { odd }} \lambda_{2} E_{i_{k}} \oplus W
$$

By Lemma 17 there is a noncompact operator

$$
T \in \mathcal{Q} \mathscr{Q}\left[\left\{\sum_{\substack{k=1 \\ k \text { odd }}}^{n} E_{i_{k}}\right\}_{n=1}^{\infty}\right] \quad \text { with } \delta(T)=0 .
$$

Let $X$ be a partial isometry with initial space $\left(\Sigma_{k \text { odd }} E_{i_{k}}\right) \mathcal{X}$ and final space $\left(\Sigma_{k \text { even }} E_{i_{k}}\right) \mathcal{H}$ such that $X E_{i_{k}} X^{*}=E_{i_{k+1}}$ for each odd $k$; note that $X T \in$ $\mathcal{Q} \mathscr{Q}(\mathcal{P})$ and is not compact so that $V(X T)-(X T) V=\left(\lambda_{1}-\lambda_{2}\right) X T \notin$ $\mathcal{C}(\mathcal{H})$. Because of this contradiction, we conclude that the essential commutant of $\mathscr{Q} \mathscr{D}(\mathscr{P})$ is $\mathbf{C} \cdot 1+\mathcal{C}(\mathcal{H})$.

We remark that Theorem 20 is particularly interesting, as recently, in [11, Corollary 1.9], it was shown that every unital, separable, $C^{*}$-subalgebra of the Calkin algebra equals its double commutant; $\pi(\mathscr{Q} \mathscr{D}(\mathscr{P}))$ is the only example known to the author of a unital $C^{*}$-subalgebra of the Calkin algebra which does not equal its double commutant.

Most of these results are also contained in the author's thesis (June 1975, University of California, Berkeley). The author is extremely grateful to Professor Calvin C. Moore for his mathematical guidance and warm encouragement. She wishes to thank Professor Marc A. Rieffel for reading her thesis and then suggesting many improvements. She wishes to acknowledge also the stimulating influence of a paper of B. Johnson and S. Parrott [8].

\section{REFERENCES}

1. W. B. Arveson, Interpolation problems in nest algebras, J. Functional Analysis 20 (1975), 208-233. MR 52 \#3979.

2. , An invitation to $C^{*}$-algebras, Graduate Texts in Math., Springer-Verlag, New York, 1976.

3. Subalgebras of $C^{*}$-algebras. II, Acta. Math. 128 1972, 271-308.

4. R. G. Douglas, Banach algebra techniques in operator theory, Academic Press, New York, 1972. MR 50 \# 14335.

5. I. C. Gohberg and M. G. Krein, Theory and applications of Volterra operators in Hilbert space, Izdat. "Nauka", Moscow, 1967; English transl., Transl. Math. Monographs, vol. 24, Amer. Math. Soc., Providence, R. I., 1970. MR 36 \# 2007; 41 \#9041.

6. P. R. Halmos, A Hilbert space problem book, Van Nostrand, Princeton, N. J., 1967. MR 34 \#8178. 
7. \#5066.

8. B. E. Johnson and S. K. Parrott, Operators commuting with a von Neumann algebra modulo the set of compact operators, J. Functional Analysis. 11 (1972), 39-61. MR 49 \#5869.

9. S. Kwapien and A. Pelczyński, The main triangle projection in matrix spaces and its applications, Studia Math. 34 (1970), 43-68. MR 42 \#5011.

10. J. Ringrose, Compact non-self-adjoint operators, Van Nostrand Reinhold, London, 1971.

11. D. Voiculescu, A non-commutative Weyl-von Neumann theorem, Rev. Roumaine Math. Pures Appl. 21 (1976), 97-113.

Department of Mathematics, Universtty of California, San Diego, la Jolla, CaljFORNIA 92093

Current address: Systems Study, 8326, Sandia Laboratories, Livermore, California 94550 